\title{
Colostrum and breast milk in the neonatal period: The benefits keep adding up
}

We all know that feeding newborns with breast milk (BM) has been occurring since time immemorial, and this is how the human race has evolved. However, after World War II, breastfeeding decreased dramatically, especially due to the effects of the technological era that led the population to falsely believe that infant formula could replace BM. In the early 1970s, in some developed countries, only $25-30 \%$ of mothers breastfed their newborns. This was an important cause for concern, especially among Societies of Pediatrics, and thanks to multiple actions, BM gradually regained ground.

In later years, many advances were made in relation to the protective aspects of BM that helped to increasingly gain knowledge on its mechanisms in newborn infants. Thus, we established an amazing variety of BM benefits for newborns, in particular for extremely preterm infants when given from their first day of life and, if possible, in an exclusive manner. ${ }^{1}$ Noteworthy benefits include the reduction of necrotizing enterocolitis (NEC), late-onset sepsis, retinopathy of prematurity (ROP), bronchopulmonary dysplasia (BPD), and readmissions in the first year of life. In addition to these benefits, in the long term, BM offers evidently improved results in neurodevelopment.

More recently, not only new studies have been conducted in BM composition, but also in the differences between the breast milk from mothers of preterm and term infants, and its administration as early as possible. Several studies observed the protective factors of BM against infections and other disorders. This is the case of lactoferrin, whose concentration is much higher in colostrum than in BM, and in the BM from mothers of preterm infants than in those of full-term babies. ${ }^{2,3}$

Colostrum's higher level of lactoferrin, together with other protective factors, account for its extraordinary relevance in the clinical course of small preterm infants.

It is worth noting that for decades, since the early 1970s, it has been possible to prove its outstanding benefits in the Maternidad Sardá of Buenos Aires through an action called "The Colostrum Plan." The Plan was launched due to the frequent epidemics of Escherichia Coli infections that caused a high mortality in the first weeks of life. A prospective study was conducted with the administration of colostrum from day one to hospitalized infants born with less than $2500 \mathrm{~g}$. The administration of $0.5 \mathrm{~mL} / \mathrm{kg} /$ day of colostrum using a nasogastric tube from the first day to 280 newborns resulted in a marked reduction of sepsis and, thereby, a significant mortality reduction. Based on our knowledge, this study published in 1974 was the first one to assess the administration of colostrum to low birth weight newborns to reduce bacterial infections. ${ }^{4}$

In recent years, colostrum given to very small preterm infants, preferably in the fauces, has attracted a lot of attention; several studies have demonstrated its substantial benefits, especially a reduction in NEC and late-onset sepsis. The decrease in NEC, which occurs in $5-10 \%$ of preterm infants with a birth weight of less than $1500 \mathrm{~g}$, probably has a greater impact because it reduces the high morbidity and mortality in the short and long term resulting from severe complications, including cholestasis, short bowel syndrome, growth deficit, and neurodevelopmental disorders. ${ }^{1,5,6}$ In addition, a reduction in late-onset sepsis among extremely preterm infants is of utmost importance due to its high mortality.

There are several mechanisms through which both colostrum and BM are beneficial and protective, not only in the neonatal period but also in the future. For example, human milk oligosaccharides (HMOs), lactoferrin, IgA, lysozyme, and bile salt-stimulated lipase. It should be noted that HMOs play highly important roles through three main functions: their action as prebiotics, the competition for pathogens, and fucose and sialic acid supply. Fucose plays an essential role by modulating the immune system, eliminating bacteria, and stimulating host defense. Sialic acid acts on synapsis function, memory formation, and cell membrane communication, all of which have an impact on infants' neurodevelopment and their future years.

Other factors that should be considered are the development of the infant microbiome, which will have implications on health and immunity for life, and the gut microbiota, which is present in the fetus, newborn infants, children, and adults. ${ }^{7}$ Currently, it is clear that the fetus does not develop in a sterile environment and, most 
probably, it is exposed to a wide variety of microbial components and live microbes.

Several data suggest that most pathogens causing late-onset sepsis come from the gut, including Gram-positive cocci. In addition, NEC and sepsis probably involve a "dysbiosis" of the gut microbiota as part of their pathophysiology.

Unfortunately, some interventions damage the newborn's gut microbiota, especially those conducted in very small preterm infants. Antibiotic indication since day one is a common, but mostly unjustified, practice. In most neonatal care units, over $80 \%$ of extremely preterm infants receive antibiotics at birth. Cesarean delivery is another unjustified intervention because it has not been demonstrated that it is better for preterm births. Both actions play an important role by altering the microbiota development, which leads to poor infant outcomes, such as NEC and late-onset sepsis. ${ }^{1,8}$

I should underline another aspect: the use of donor milk, which is of great value when the mother's milk supply is insufficient or unavailable. Although there is controversy about whether donor milk has the same effect as BM, several studies have observed very similar effects, so it is better to use donor milk, which yields markedly superior results, then formula. ${ }^{9}$

Other benefits of BM in very small preterm infants have been published. A recent study observed a reduction in BPD associated with the amount of BM from birth to 36 weeks old. That is to say, the greater the amount of BM, the lower the risks for $\mathrm{BPD} .{ }^{10}$ In addition, three recent metaanalyses showed that any amount of human milk is strongly associated with the protection against ROP. One of these meta-analyses showed a significant $60 \%$ reduction in severe ROP with a greater exposure to human milk, whereas lower oxygen saturation targets were associated with only a $42 \%$ lower risk for ROP. ${ }^{11}$

Finally, it is necessary to point out that all the benefits of colostrum and BM for very small preterm infants are only possible if we become involved in breastfeeding. We must accept that it is essential to stimulate mothers before delivery, and most of all, to encourage those who give birth prematurely so that they become aware of the great importance of breastfeeding for a better clinical course of their infants in the short and long term. Also, in neonatal care units, it is imperative to strive to have mothers breastfeeding their infants at the time of discharge from the unit.

\section{José M. Ceriani Cernadas} Editor-in-chief

http:/ / dx.doi.org/10.5546/ aap.2018.eng.234

To cite: Ceriani Cernadas JM. Colostrum and breast milk in the neonatal period: The benefits keep adding up. Arch Argent Pediatr 2018;116(3):234-235.

\section{REFERENCES}

1. Underwood MA. Human milk for the premature infant. Pediatr Clin North Am 2013; 60(1):189-207.

2. Ronayne de Ferrer PA, Baroni A, Sambucetti ME, et al. Lactoferrin levels in term and preterm milk. J Am Coll Nutr 2000; 19(3):370-3.

3. Turin CG, Zea-Vera A, Rueda MS, et al. Lactoferrin concentration in breast milk of mothers of low-birth-weight newborns. J Perinatol 2017; 37(5):507-12.

4. Larguía AM, Urman J, Ceriani Cernadas JM, et al. Inmunidad local en el recién nacido. Primera experiencia con la administración de calostro humano a recién nacidos pretérmino. Arch Argent Pediatr 1974; 72(5):109-25.

5. Gephart SM, Weller M. Colostrum as oral immune therapy to promote neonatal health. Adv Neonatal Care 2014; 14(1):44-51.

6. Meinzen-Derr J, Poindexter B, Wrage L, et al. Role of human milkin extremely low birth weightinfants' risk of necrotizing enterocolitis or death. J Perinatol 2009; 29(1):57-62.

7. Pannaraj PS, Li F, Cerini C, et al. Association between breast milk bacterial communities and establishment and development of the infant gut microbiome. JAMA Pediatr 2017; 171(7):647-54.

8. Alexander VN, Northrup V, Bizzarro MJ. Antibiotic exposure in the newborn intensive care unit and the risk of necrotizing enterocolitis. J Pediatr 2011; 159(3):392-7.

9. de Halleux V, Pieltain C, Senterre T, et al. Use of donor milk in the neonatal intensive care unit. Semin Fetal Neonatal Med 2017; 22(1):23-9.

10. Patel AL, Johnson TJ, Robin B, et al. Influence of own mother's milk on bronchopulmonary dysplasia and costs. Arch Dis Child Fetal Neonatal Ed 2017; 102(3):F256-F261.

11. Fang JL, Sorita A, Carey WA, et al. Interventions to prevent retinopathy of prematurity: a meta-analysis. Pediatrics 2016; 137(4):e20153387. 